\title{
Early Midregional pro-Atrial Natriuretic Peptide predicts the occurrence of atrial fibrillation in stroke patients: Data from the BIOSIGNAL Study
}

J. Schweizer ${ }^{1}$, K. Spanaus ${ }^{2}$, M. Arnold ${ }^{3}$, U. Fischer ${ }^{3}$, L. H. Bonati ${ }^{4}$, D. De Marchis ${ }^{4}$, T. Kahles ${ }^{5}$, K. Nedeltchev $^{5}$, C. Cereda $^{6}$, G. Kägi $^{7}$,

A. Bustamante ${ }^{8}$, J. Montaner ${ }^{8}$, G. Ntaios ${ }^{9}$, C. Foerch ${ }^{10}$, P Michel ${ }^{11}$, A. Luft ${ }^{1}$, A. von Eckardstein ${ }^{2}$, M. Katan ${ }^{1}$ for the BIOSIGNAL investigators

Background

- The use of rapidly measurable blood biomarkers may advance the identification of patients with underlying atrial cardiopathy, who: may benefit more from direct oral anticoagulants compared to antiplatelets for secondary prevention.

- The objective of this study was to assess the incremental value of midregional pro-Atrial Natriuretic Peptide (MR-proANP) in : identifying cardio-embolic stroke (CE stroke according to the TOAST criteria), newly diagnosed atrial fibrillation (AF) during: hospitalization and on follow-up as well as recurrent vascular events after ischemic stroke.

\section{Methods}

- In this prospective, multicenter cohort study (2014-2017) we measured MR-pro ANP in ischemic stroke patients within $24 \mathrm{~h}$ of symptoms onset. (By a sandwich immunoassay (BRAHMS AG, Berlin).

- Univariate and multivariate logistic regression models were fitted to estimate odds ratios for the magnitude and independence of the association of MR-proANP with each outcome measure.

\section{Outcome measures}

- CE stroke defined according to the Trial of Org 10172 in Acute Stroke Treatment (TOAST) criteria.

- AF was assessed- during hospitalization (at least 48 hours ECG) and 3 \& 12 months after stroke (at least 7 days of ECG monitoring). AF was defined as at least 30 seconds of atrial arrhythmia.

- Composite outcome of recurrent stroke (including fatal stroke) and other cardiovascular death within 365 days after the index stroke.

\section{Results}

\begin{tabular}{|c|c|c|}
\hline \multicolumn{3}{|l|}{ Baseline } \\
\hline $\mathbf{N}(\%)$ & 1767 & \\
\hline \multicolumn{3}{|l|}{ Demographic data } \\
\hline Age, median (IQR), & 74.4 & $(63.8-82)$ \\
\hline Women, $n(\%)$ & 739 & $(42)$ \\
\hline \multicolumn{3}{|l|}{ Medical history, $\boldsymbol{n}(\%)$} \\
\hline Hypertension & 1287 & (73) \\
\hline Diabetes mellitus & 299 & $(17)$ \\
\hline Smoking & 398 & (23) \\
\hline Dyslipidemia & 1206 & (68) \\
\hline History of atrial fibrillation & 325 & $(18)$ \\
\hline Chronic heart failure & 111 & (6) \\
\hline Myocardial infarction & 173 & $(10)$ \\
\hline Previous stroke & 304 & $(17)$ \\
\hline $\begin{array}{l}\text { Modified Charlson Index (comorbidity } \\
\text { score points) }\end{array}$ & 1 & $(0-2)$ \\
\hline \multicolumn{3}{|l|}{ Clinical data, median (IQR) } \\
\hline NIHSS on admission (points) & 5 & $(2-11)$ \\
\hline \multicolumn{3}{|l|}{ Treatment of stroke, $n(\%)$} \\
\hline IV thrombolysis & 708 & $(40)$ \\
\hline IA thrombolysis & 142 & (8) \\
\hline Thrombectomy & 335 & (19) \\
\hline \multicolumn{3}{|l|}{ Laboratory values, median (IQR) } \\
\hline MR-proANP (pmol/l) & 145.1 & $(83.8-248.9)$ \\
\hline Creatinine (umol/l) & 83 & $(69-99)$ \\
\hline \multicolumn{3}{|l|}{ Stroke etiology (TOAST) , $n(\%)^{a}$} \\
\hline Large vessel stroke & 259 & $(15)$ \\
\hline Cardioembolic stroke & 587 & (33) \\
\hline Microangiopathic stroke & 183 & $(10)$ \\
\hline Other known & 94 & (5) \\
\hline Unknown /cryptogenic stroke & 648 & $(37)$ \\
\hline $\begin{array}{l}\text { Abbreviations: } N=\text { number, } I Q R=\text { interquartile range; } N \\
\text { Stroke Scale; IA = intra-arterial; IV intravenous; } \mathrm{MR}=\mathrm{M} \\
\text { weighted imaging; TOAST = Trial of Org } 10172 \text { in Acut } \\
\text { midregional proatrial natriuretic peptide. Statistics: value } \\
\text { (number). aBecause of rounding, percentages may not t }\end{array}$ & $\begin{array}{l}\text { SS }=\text { Natic } \\
\text { gnetic Res } \\
\text { Stroke Trea } \\
\text { are media } \\
\text { al } 100 \text {. }\end{array}$ & $\begin{array}{l}\text { I Institutes of Health } \\
\text { ance; DWI = diffusion- } \\
\text { ent; MR-proANP = } \\
\text { IQR) or percent }\end{array}$ \\
\hline
\end{tabular}

\begin{tabular}{|c|c|c|c|c|c|}
\hline & Predictors & OR $^{a}$ & & $5 \% \mathrm{Cl}$ & $\begin{array}{l}P \text { - } \\
\text { Value }\end{array}$ \\
\hline \multirow{11}{*}{$\begin{array}{l}\text { Cardio - } \\
\text { embolic } \\
\text { stroke }\end{array}$} & Age & 0.96 & 0.95 & -0.98 & $<0.01$ \\
\hline & Women & 0.83 & 0.57 & -1.21 & 0.35 \\
\hline & History of AF & 10.84 & 6.35 & -18.5 & $<0.01$ \\
\hline & Smoking & 0.67 & 0.43 & -1.03 & 0.07 \\
\hline & Chronic Heart failure & 1.87 & 0.83 & -4.22 & 0.13 \\
\hline & Hyperlipidemia & 0.62 & 0.43 & -0.90 & 0.01 \\
\hline & NIHSS & 1.01 & 0.98 & -1.04 & 0.40 \\
\hline & MR-proANP & 4.06 & 2.85 & -5.79 & $<0.01$ \\
\hline & Creatinine & 0.73 & 0.42 & -1.29 & 0.28 \\
\hline & Antihypertensive drugs & 0.92 & 0.61 & -1.36 & 0.66 \\
\hline & sion size & 0.83 & 0.57 & -1.22 & 0.35 \\
\hline \multirow{7}{*}{$\begin{array}{l}\text { Newly } \\
\text { detected } \\
\text { AF }\end{array}$} & Age & 1.00 & 0.99 & -1.02 & 0.66 \\
\hline & Women & 1.18 & 0.79 & -1.76 & 0.43 \\
\hline & NIHSS & 1.01 & 0.98 & -1.04 & 0.60 \\
\hline & MR-proANP & 3.28 & 2.28 & -4.73 & $<0.01$ \\
\hline & Creatinine & 0.77 & 0.43 & -1.38 & 0.38 \\
\hline & Small lesion size & 0.60 & 0.39 & -0.94 & 0.03 \\
\hline & & $\mathbf{H R}^{\mathbf{a}}$ & \multicolumn{2}{|c|}{$95 \% \mathrm{Cl}$} & $\begin{array}{l}\text { P- } \\
\text { Value }\end{array}$ \\
\hline \multirow{6}{*}{$\begin{array}{l}\text { Recurrent } \\
\text { ischemic } \\
\text { event }\end{array}$} & Age & 1.02 & 1.00 & -1.03 & $<0.01$ \\
\hline & Women & 1.15 & 0.90 & -1.46 & 0.26 \\
\hline & $\begin{array}{l}\text { Previous } \\
\text { cerebrovascular event }\end{array}$ & 1.53 & 1.16 & -2.01 & $<0.01$ \\
\hline & NIHSS & 1.07 & 1.06 & -1.09 & $<0.01$ \\
\hline & MR-proANP & 1.28 & 1.02 & -1.54 & 0.03 \\
\hline & Charlson- Index & 1.07 & 1.00 & -1.14 & 0.04 \\
\hline
\end{tabular}

Abbreviations: $\mathrm{Af}=$ Atrial fibrillation $\mathrm{Cl}=$ confidence interval; resonance; MR-proANP $=$ midregional

proatrial natriuretic peptide; NA = not applicable; NIHSS on admission= NIH Stroke Scale; OR = odds ratio, $\mathrm{HR}=$ hatard ratio; AS5F score to detect paroxysmal atrial fibrillation after stroke including: age $x$ 0.76 points/year, stroke Severity NIH-SS $\leq 5=9$ points, NIHSS $>5=21$ points

a ORs and hazard ratio refer to a 1-unit increase in the explanatory variable and to any 10 -fold increase in MR-proANP and Creatinine (log transformed with a base of 10).

\section{Conclusions}

MR-proANP is an independent marker for cardio-embolic stroke etiology according to the TOAST criteria, newly detected atrial fibrillation and cardiovascular events up to one year after the index stroke. Therefore MR-proANP seems to be a good candidate to select patients for prolonged rhythm monitoring in clinical routine or even for oral anticoagulation in a randomized controlled trial. 